\title{
In-house ELISA screening using a locally-isolated Leptospira in Malaysia: determination of its cut-off points
}

Xue Ting Tan ${ }^{1 *}$, Fairuz Amran ${ }^{1}$, Kee Chee Cheong $^{2}$ and Norazah Ahmad ${ }^{1}$

\begin{abstract}
Background: Leptospirosis is a zoonotic disease caused by Leptospira species and is distributed globally. Microscopic agglutination test (MAT) is the serological 'gold standard' for diagnosis of leptospirosis but it is time-consuming and labour-intensive. An alternative serological method that is rapid, sensitive and specific is important for early treatment to reduce morbidity and mortality. The use of local Leptospira isolation may improve the sensitivity and specificity of the test because it may varies from one geographical region to another region. The objective of this study was to determine the sensitivity, specificity and cut-off points for an in-house Immunoglobulin M (IgM) enzyme-linked immunosorbent assay (ELISA) using a locally isolated Leptospiral strain IMR/175 as the antigen for the detection of anti-Leptospiral IgM.
\end{abstract}

Methods: Serum samples from 270 patients with clinical symptoms of leptospirosis were subjected to the in-house IgM ELISA, MAT and Leptospirosis rapid test. The optimal cut-off values for positivity and negativity of the IgM ELISA were determined by Receiver Operating Characteristic curves and mean \pm 2 standard deviation (SD) analyses of the ELISA values.

Results: The area under the curve (AUC) which indicates the diagnostic performance of the in-house IgM ELISA was 0.953 (95\% Confidence Interval, Cl: $0.928,0.978$ ). The sensitivity and specificity of $90.38 \%$ and $87.72 \%$ respectively were obtained with the cut-off point of 0.55 . A higher sensitivity (96.15\%) was obtained when the cut-off point was set at 0.45 .

Conclusions: The in-house IgM ELISA assay using local Leptospira isolation was shown to be sensitive and may be suitable to use for the serological diagnosis of leptospirosis for our local hospital setting.

Keywords: In-house ELISA, Leptospirosis, Malaysia, Cut-off points

\section{Background}

Leptospirosis is an acute febrile disease with worldwide distribution [1,2]. It is a problem of developing countries due to humid tropical and subtropical weather. It is identified as a re-emerging infectious disease since large outbreaks have occurred in many countries including Malaysia [3]. The overall incidence of leptospirosis in Malaysia was $13 \%$ [4] with fatality percentage is estimated around 10\% [5].

Most of the human cases of leptospirosis are diagnosed by serological methods. The reference standard

\footnotetext{
* Correspondence: tanxt@imr.gov.my

'Bacteriology Unit, Infectious Disease Research Centre, Institute for Medical Research, 50588, Jalan Pahang, Kuala Lumpur, Malaysia

Full list of author information is available at the end of the article
}

for serological test is the microscopic agglutination test (MAT) [5]. However, MAT has many disadvantages including time-consuming, tedious maintenance of live Leptospira, risk of cross-contaminations and hazardous to workers because of the exposure to live bacteria [6]. Besides that, MAT may not be useful for acute patient management because of the extensive technical resources requirements and is also not applicable in some routine diagnostic laboratory setting [7].

By comparison, the Immunoglobulin M (IgM) enzymelinked immunosorbent assay (ELISA) is relatively cheaper than MAT and is more sensitive because of IgM can be detected earlier $[6,8]$. This is important because treatment 
can be administered in time thus preventing the progression of the disease into severe stage.

Most of the commercially available ELISA kits using non-pathogenic L. biflexa strain patoc $I$ as the antigen [6,9-11]. Since the prevalence of Leptospiral serogroups varies geographically [12], we developed an in-house ELISA using a locally isolated Leptospira strain IMR/175 as the antigen for the detection of Leptospira IgM antibodies among patients in Malaysia. To our knowledge, this is the first study about in-house ELISA on detecting Leptospirosis in Malaysia. The aim of this study was to determine the cut-off points of the in-house ELISA and their accuracy, sensitivity and specificity.

\section{Methods}

\section{Ethics statement}

The study protocol which included participants providing written consent prior to the study, was approved by Malaysia Research \& Ethics Committee, Ministry of Health Malaysia, Malaysia.

\section{Serum samples}

A total of 270 sera received from patients presented with symptoms suggestive of leptospirosis during the year 2012 were subjected to a commercially available leptospirosis rapid test kit Leptorapid ${ }^{\circ}$, MAT, and in-house IgM ELISA in our laboratory. The usual presentations of the patients were acute febrile illness, headache, myalgia, jaundice, cough, vomit, abdominal pain, diarrhea and haemorrhages. The sample size was calculated based on expected sensitivity of $85 \%$, specificity of $90 \%$, precision of 0.10 and $95 \%$ of confidence level $[4,13]$.

\section{Leptospirosis rapid test}

The rapid test was performed according to the instruction of Leptorapide ${ }^{\circ}$ (Linnodee Ltd, Northern Ireland). Briefly, about $5 \mu \mathrm{l}$ of each leptorapide suspension and test sera were mixed on the agglutination card. The agglutination was examined within three minutes and the results were interpreted using a score card provided by manufacturer.

\section{MAT}

The MAT was performed as described by the World Health Organization [2]. Briefly, live Leptospira cell suspensions from 20 Leptospiral serovars were added to a two-fold serially diluted serum in 96-well U-bottomed microtiter plates and were incubated at room temperature for 2 hours. The panel of leptospires consisted of IMR/1, IMR/22, IMR/27, IMR/115, IMR, 175, IMR/803, Australis, Autumnalis, Bataviae, Canicola, Celledoni. Grippotyphosa, Hardjoprajitno, Icterohaemorrhagiae, Javanica, Pyrogenes, Tarassovi, Djasiman, Patoc and Pomona.
Micro-agglutination was examined by dark-field microscopy. The titer was calculated as the reciprocal of the highest dilution of serum which showed at least $50 \%$ of agglutination of the Leptospiral cells. Cases were defined as positive for leptospirosis if agglutination titer achieved $\geq 400$ level to one or more Leptospira serovars. All samples were screened by MAT to determine the serum is positive or negative for leptospirosis.

\section{IgM ELISA}

The antigen was prepared from a nonpathogenic strain IMR/175 which was isolated from a water sample in a pond from Sarawak, Malaysia. The antigen was diluted 1:2 in phosphate buffered saline (PBS) $\mathrm{pH}$ 7.2. The protocol of preparing antigen and coating plate was according to the protocol described by Goris et al. [14]. Briefly, the culture was grow in EMJH medium, killed by formalin (final concentration $0.2 \% \mathrm{v} / \mathrm{v}$ ), heated in a boiling water bath for $30 \mathrm{~min}$ and centrifuged for $30 \mathrm{~min}$ at $10,000 \mathrm{~g}$. The $100 \mu \mathrm{l}$ supernatant was pipetted into the well and left to be evaporated. Before the plate being used, it was washed twice with PBS containing 0.05\% Tween 20. Serial serum dilutions of 80 and 160 were added into the coated plates and incubated for $30 \mathrm{~min}$ at $37^{\circ} \mathrm{C}$. The plate was then washed again and subsequently $100 \mu \mathrm{l}$ of Horseradish peroxidase-conjugated anti-human IgM (KPL) was added to the plate. After $30 \mathrm{~min}$ incubation at $37^{\circ} \mathrm{C}$, the plate was washed again. Next, $100 \mu \mathrm{l}$ of substrate (5-aminosalicyclic acid) was added and the plate incubated for one hour at room temperature. The absorbance of the suspensions was read at $490 \mathrm{~nm}$ using Opsys $\mathrm{Mr}^{\mathrm{TM}}$ Microplate reader (Dynex Technologies). The sample was tested in duplicates and an average optical density was obtained for each sample. Each reading was recorded and statistically analysed as mentioned below.

\section{Statistical analysis}

All statistical analysis were performed with the statistical software package PASW statistics 18.0 for windows (SPSS Inc.). Receiver operating characteristic (ROC) curves were used to determine the optimal level of the IgM ELISA test for serum dilutions of 1:80 and 1:160. The sensitivities and specificities of IgM ELISA for diagnosis of leptospirosis (positive MAT) were also demonstrated at different cutoff values. In addition, the positive predictive value (PPV) and negative predictive value (NPV) were also calculated at different cut-off values.

Sensitivity is defined as the probability of correctly identifying those with positive leptospirosis by MAT for a given ELISA cut-off value. Specificity is defined as the probability of correctly identifying those with negative leptospirosis by MAT at a given IgM ELISA cut-off value. PPV is the probability that subjects with a positive ELISA test truly have leptospirosis while NPV is the 
probability that subjects with a negative ELISA test truly do not have leptospirosis.

The optimal IgM ELISA cut-off value was determined by comparing the mean \pm 2 standard deviation (SD) of IgM ELISA value for both positive and negative MAT samples. The area under the curve (AUC) with 95\% confidence intervals was used to compare the predictive capability of the two IgM ELISA dilutions for identification of those diagnosed by MAT. Higher of the values of AUC indicates a higher predictive capability of IgM ELISA in diagnosing leptospirosis. For all analyses, $p$ values less than 0.05 were considered as statistically significant.

\section{Results and discussion}

Out of 270 samples tested, 114 samples were negative while 156 were positive by both MAT and rapid test. The samples were further tested by in-house ELISA and all the obtained data were used for ROC curve analysis to determine the cutoff point from optimal sensitivity and specificity achieved in the assay.

The AUC reflects how good of the test to distinguish between patients with and without disease [14]. In the ROC curve analysis, it showed that the AUC for serum dilutions 1:80 and 1:160 were 0.953 (95\% Confidence Interval, $\mathrm{CI}=0.928-0.978)$ and 0.927 (95\% CI $=0.896-0.958)$ respectively (Figures 1 and 2). Although the diagnostic performances of our in-house IgM ELISA were high for both dilutions, the 1:80 dilution had better diagnostic

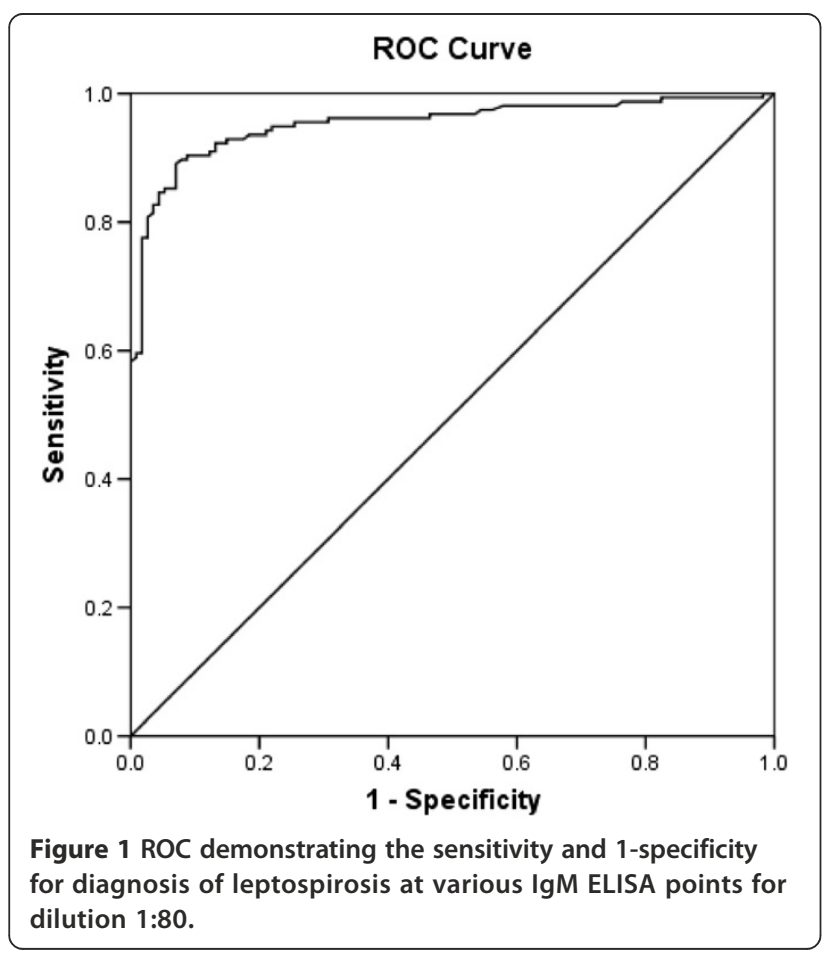

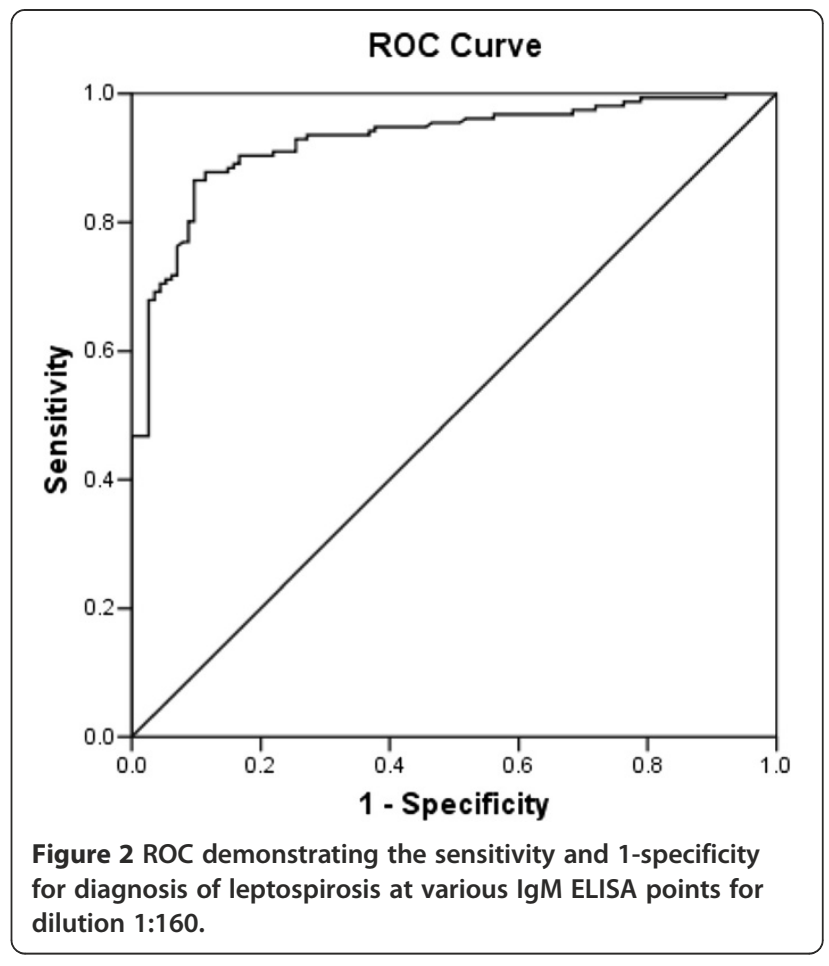

performance than 1:160. Thus, we decided to select cutoff points from the 1:80 dilution.

The mean $\pm 2 \mathrm{SD}$ value for both positive and negative MAT samples of dilutions are listed in Table 1. To determine the negative and positive cut-off points, we referred to the value of mean-2SD of positive MAT samples and mean $+2 \mathrm{SD}$ of negative MAT samples respectively. After that, the sensitivity and specificity of yielded cut- off values from ROC curves analysis were compared (Table 2). From the analysis of mean $\pm 2 \mathrm{SD}$ of the 1:80 dilution, cut-off values of 0.45 and 0.64 were obtained. However, 0.64 was not selected as our positive cut-off point because it gave sensitivity of less than $90 \%$. As the leptospirosis incidence and fatality of Leptospirosis in Malaysia is high, a very sensitive test is needed because it is very important not to miss a diagnosis [15]. We decided the sensitivity of the positive cut-off shall be equal or greater than $90 \%$, thus 0.45 and 0.55 were selected as negative and positive cut-off points respectively. The value between 0.45 and 0.55 is considered intermediate level

Table 1 Statistical values of both positive and negative MAT samples for ELISA with serum dilutions of 1:80

\begin{tabular}{lll}
\hline Statistical value & Positive MAT samples & Negative MAT samples \\
\hline Mean & 0.7783 & 0.4316 \\
SD & 0.1627 & 0.1057 \\
Mean + 2 SD & 1.1037 & 0.6430 \\
Mean-2 SD & 0.4529 & 0.2202 \\
\hline
\end{tabular}


Table 2 Sensitivity and specificity of IgM ELISA for dilution 1:80 and 1:160 at different cut-off values

\begin{tabular}{llllll}
\hline Dilution & $\begin{array}{l}\text { Cut-off } \\
\text { value }\end{array}$ & Sensitivity (\%) & Specificity (\%) & $\begin{array}{l}\text { Positive } \\
\text { predictive } \\
\text { value (\%) }\end{array}$ & $\begin{array}{l}\text { Negative } \\
\text { predictive } \\
\text { value (\%) }\end{array}$ \\
\hline \multirow{4}{*}{$1: 80$} & 0.45 & 96.15 & 57.89 & 75.76 & 91.67 \\
& 0.50 & 94.87 & 75.44 & 84.09 & 91.49 \\
& 0.55 & 90.38 & 87.72 & 86.96 & 90.97 \\
& 0.60 & 85.26 & 93.86 & 95.00 & 82.31 \\
$1: 160$ & 0.65 & 80.13 & 97.37 & 97.66 & 78.17 \\
\hline & 0.45 & 88.46 & 84.21 & 88.46 & 84.21 \\
& 0.50 & 83.33 & 90.35 & 92.20 & 79.84 \\
& 0.55 & 72.44 & 92.98 & 93.39 & 71.14 \\
& 0.60 & 66.67 & 97.37 & 97.20 & 68.10 \\
& 0.65 & 57.05 & 97.37 & 96.74 & 62.36 \\
\hline
\end{tabular}

and it is recommended to be repeated with follow-up sample.

To emphasize, our institute is the main institute that carry out the leptospirosis routine diagnosis work which submitted from clinics and hospitals throughout the country [16]. It is important to note that about $50 \%$ of the positive sera were agglutinated with this strain in our MAT test yearly. We had submitted the partial sequence of $16 \mathrm{~S}$ ribosomal RNA gene of this strain to NCBI (GeneBank: JX292159) and research on this strain is still ongoing.

As convalescent-phase serum is hardly available in Malaysia hospital, it is difficult to determine the fourfold or greater rise in MAT titers between acute- and convalescent-phase which define a leptospirosis case. Thus, we referred the guidelines given by Ministry of Health Malaysia [17]. The case was defined positive if single serum titre was equal to or greater than 400 while fourfold or greater rise in MAT titre if paired sera were obtained. The same case definition also used in other studies [11,18-20]. Besides, the case also can be defined positive if MAT is greater than or equal to 400 and supported by a positive IgM result [21]. In this study, we had further increased the reliability of our result with including the samples which showed positive from both rapid and MAT tests. Lastly, further study on more clinical samples is necessary to determine the usefulness of our IgM ELISA.

\section{Conclusions}

This study showed that using the cut-off points determined from 1:80 dilution in our in-house ELISA using local Leptospira isolation is sensitive for the detection of leptospirosis cases when compared to the MAT results. The positive and negative cut-off points for this IgM ELISA are 0.55 and 0.45 respectively.

\section{Abbreviations}

AUC: Area under the curve; Cl: Confidence interval; ELISA: Enzyme-linked immunosorbent assay; IgM: Immunoglobulin M; MAT: Microscopic agglutination test; PBS: Phosphate buffered saline; ROC: Receiver operating characteristic; PPV: Positive predictive value; NPV: Negative predictive value; SD: Standard deviation.

\section{Competing interests}

The authors declare that they have no competing interests.

\section{Authors' contributions}

$\mathrm{XTT}$ carried out the study, participated in the statistical analysis and drafted the manuscript. KCC performed the statistical analysis, interpretation of the data and drafted the manuscript. FA conceived the study, participated in study design and critical revision for important intellectual content. NA critically revised the manuscript and provided general support. All authors read and approved the final manuscript.

\section{Acknowledgments}

The authors would like to thank the Director General of Health, Ministry of Health Malaysia for permission to publish this article. The authors would also like to express their appreciation to the Director of the Institute for Medical Research for her support in this study.

\section{Author details}

${ }^{1}$ Bacteriology Unit, Infectious Disease Research Centre, Institute for Medical Research, 50588, Jalan Pahang, Kuala Lumpur, Malaysia. ${ }^{2}$ Epidemiology and Biostatistics Unit, Medical Research Resource Centre, Institute for Medical Research, 50588, Jalan Pahang, Kuala Lumpur, Malaysia.

Received: 8 July 2014 Accepted: 14 October 2014

Published online: 23 October 2014

\section{References}

1. Levett PN: Usefulness of serologic analysis as a predictor of the infecting serovar in patients with severe leptospirosis. Clin linfect Dis 2003, 36:447-452.

2. World Health Organization: Human leptospirosis: guidance for diagnosis, surveillance and control. 2003, http://www.who.int/csr/don/en/ WHO_CDS_CSR_EPH_2002.23.pdf.

3. Zavitsanou A, Babatsikou F: Leptospirosis: epidemiology and preventive measures. Health Sci J 2008, 2(2):75-82.

4. Lim JK, Murugaiyah VA, Ramli A, Abdul Rahman H, Mohamed N, Shamsudin N, Tan JC: A case study: leptospirosis in Malaysia. WebmedCentral linfect Dis 2011, 2(12):WMC002764.

5. Lim VKE: Leptospirosis: a re-emerging infection. Malayas J Pathology 2011, 33(1):1-5.

6. Ahmad SN, Shah S, Ahmad FMS: Laboratory diagnosis of leptospirosis. J Postgrad Med 2005, 51:195-200.

7. Levett PN: Leptospirosis. Clin Microbiol Rev 2001, 14(2):296-326.

8. Desakorn V, Wuthiekanun V, Thanachartwet V, Sahassananda D, Chierakul W, Apiwattanaporn A, Day NP, Limmathurosakul D, Peacock SJ: Accuracy of a commercial IgM ELISA for the diagnosis of human leptospirosis in Thailand. Am J Trop Med Hyg 2012, 86(3):524-527.

9. Levett PN, Branch SL, Whittington CU, Edwards CN, Paxton H: Two methods for rapid serological diagnosis of acute leptospirosis. Clin Diagn Lab Immun 2001, 8(2):349-351.

10. Sambasiva RR, Naveen G, Bhalla P, Agarwal SK: Leptospirosis in India and the rest of the world. Braz J linfect Dis 2003, 7(3):178-193.

11. Musso D, Scola BL: Laboratory diagnosis of leptospirosis: a challenge. J Microbiol Immunol 2013, 46:245-252.

12. Effler PV, Bogard AK, Domen HY, Katz AR, Higa HY, Sasaki DM: Evaluation of eight rapid screening tests for acute leptospirosis in Hawaii. J Clin Microbiol 2002, 40(4):1464-1469.

13. Naing L: Sample size calculator for sensitivity and specificity studies. http://www.kck.usm.my/ppsg/stats_resources.htm.

14. Goris MGA, Leeflang MMG, Boer KR, Goeijenbier ECM Van G, Wagenaar JFP, Hartskeerl RA: Establishment of valid laboratory case definition for human leptospirosis. J Bacteriol Parasitol 2012, 3(2):1000132.

15. Anthony $\mathrm{K}$ : Understanding diagnostic tests 3: receiver operating characteristic curves. Acta Paediatr 2007, 96(5):644-647. 
16. El Jalii IM, Bahaman A: A review of human leptospirosis in Malaysia. Trop Biomed 2004, 21(2):113-119.

17. Ministry of Health Malaysia: Guidelines for the Diagnosis, Management, Prevention and Control of Leptospirosis in Malaysia. 1st edition. ; 2011. http://www.jknselangor.moh.gov.my/documents/pdf/sharingDoc/ kawalan_penyakit_berjangkit/quidelines/Leptospirosis.pdf.

18. Wuthiekanum V, Sirisukkarn N, Daengsupa P, Sakaraserane P, Sangkakam A, Chierakul W, Smythe LD, Symonds ML, Dohnt MF, Slack AT, Day NP,

Peacock SJ: Clinical diagnosis and geographic distribution of leptospiroses. Thailand Emerg Infect Dis 2007, 13(1):124-126.

19. Pradutkanchana S, Pradutkanchana J, Khuntikij P: Detection of IgM specific antibody using indirect immunofluorescent assay for diagnosis of acute leptospirosis. J Med Assoc Thai 2003, 86:641-646.

20. Appassakij H, Silpapojakul K, Wansit R, Woodtayakorn J: Evaluation of the immunofluorescent antibody test for the diagnosis of human leptospirosis. Am J Trop Med Hyg 1995, 52:340-343.

21. Queensland Government: Leptospirosis: Queensland Health Guidelines for Public Health Units. http://www.health.qld.gov.au/cdcg/index/lepto.asp.

doi:10.1186/s12879-014-0563-7

Cite this article as: Tan et al: In-house ELISA screening using a locallyisolated Leptospira in Malaysia: determination of its cut-off points. BMC Infectious Diseases 2014 14:563.

\section{Submit your next manuscript to BioMed Central and take full advantage of:}

- Convenient online submission

- Thorough peer review

- No space constraints or color figure charges

- Immediate publication on acceptance

- Inclusion in PubMed, CAS, Scopus and Google Scholar

- Research which is freely available for redistribution 